\title{
NUMERICAL INVESTIGATION OF SLOT VARIATIONS ON THE EFFICIENCY OF TANGENTIAL BLOWING AT A VERTICAL TAILPLANE WITH INFINITE SPAN
}

\author{
A. Gebhardt, German Aerospace Center (DLR), Aerodynamics and Flow Technology, \\ Lilienthalplatz 7, 38108 Braunschweig, Germany, \\ e-mail: anna.gebhardt@dlr.de, Tel.: 0049 (0)531 2952267 \\ J. Kirz, German Aerospace Center (DLR), Aerodynamics and Flow Technology, \\ Lilienthalplatz 7, 38108 Braunschweig, Germany
}

\begin{abstract}
On a swept vertical tailplane with infinite span tangential blowing over the shoulder of a deflected rudder is applied. For large rudder deflection angles the flow on the rudder is separated without blowing. A numerical study is conducted with the aim to increase the side force coefficient. This could for example be required during take-off if the engine on one side fails, necessitating the compensation of a large yawing moment. If this criterion is critical for the sizing of the vertical tailplane, active flow control like tangential blowing could help to reduce the size of the vertical tailplane and thus save weight and fuel. With a continuous slot it is demonstrated that the separation on the rudder can be reduced or avoided. It is shown that by using discrete slots this can be achieved with a smaller momentum coefficient. To analyze the effects of the discrete slots and their jets on each other and their effectivity with regard to the gain in side force coefficient a parameter study is conducted. The number of slots as well as the size of the slots in spanwise direction is varied and the impact of jet velocity changes is also studied. In comparing the results for a constant increase in side force and constant slot size in spanwise direction the configuration with the smaller number of slots but a higher jet velocity proved to be the most effective one.
\end{abstract}

Keywords: Active Flow Control, CFD, tangential blowing, Vertical Tailplane, RANS

\section{NOMENCLATURE}

AFC

DLR

\section{LUSGS}

OEI

RANS

SARC

VTP

2D

3D

$\mathrm{A}_{\text {ref }}$

$\mathrm{Aj}$

C

$\mathrm{C}_{\mathrm{fX}}$

$\mathrm{C}_{\mathrm{Y}}$

$\mathrm{C}_{\mu}$

DC

g

$\mathrm{h}_{\text {Slot }}$

$\mathrm{l}_{\text {Slot }}$

$\mathrm{M}$

$n k_{j}$

$\mathrm{N}$ correction
Active Flow Control

Deutsches Zentrum für Luft- und Raumfahrt (German Aerospace Center) Lower-Upper Symmetric Gauss-Seidel One-engine-inoperative

Reynolds-averaged Navier-Stokes [equations]

Spalart and Allmaras turbulence model with vortical and rotational flow

Vertical tailplane

Two dimensional

Three dimensional

Reference area $\left[\mathrm{m}^{2}\right]$

Area of the slot orifice $\left[\mathrm{m}^{2}\right]$

Chord length [m]

Skin friction coefficient [-]

Side force coefficient [-]

Momentum coefficient [-]

Duty cycle [-]

Gap size between slots [m]

Slot height [m]

Slot length in chord direction [m]

Mach number [-]

Mass flow rate of the jet $[\mathrm{kg} / \mathrm{s}]$

Number of slots per calculated span [-]

$\begin{array}{ll}v_{j} & \text { Jet velocity [m/s] } \\ v_{\infty} & \text { Reference onset flow velocity }[\mathrm{m} / \mathrm{s}] \\ \mathrm{w} & \text { Slot width in spanwise direction }[\mathrm{m}] \\ \mathrm{y}^{+} & \text {Dimensionless wall distance [-] } \\ \phi & \text { Sweep angle [deg] } \\ \rho_{j} & \text { Jet density }\left[\mathrm{kg} / \mathrm{m}^{3}\right] \\ \rho_{\infty} & \text { Reference onset flow density }\left[\mathrm{kg} / \mathrm{m}^{3}\right] \\ \omega_{\mathrm{x}} & \text { Rotation direction in x-direction }[1 / \mathrm{rad}]\end{array}$

\section{INTRODUCTION}

The use of active flow control is investigated for a vertical tailplane (VTP) geometry as one of the promising applications of this technology. The vertical tailplane of a transport aircraft is required for stability and control of the aircraft about the yaw axis. One case determining the size of the VTP is the failure of the critical engine. In the one-engine inoperative (OEI) condition for a conventional aircraft with the engines installed on the left and right wing, the resulting asymmetric thrust creates a moment around the yaw axis. This must be counteracted by the VTP. Here take-off is a critical flight segment where the airspeed is relatively low and thus also the aerodynamic forces produced by the VTP, but the thrust of the engines is high. This leads to a large yawing moment created by the still running engine which has to 
be compensated by the VTP. On the other hand, in cruise flight, the VTP is larger than necessary to satisfy cruise stability requirements for modern transport aircraft with electronic flight control systems due to some "artificial" stability contribution which such systems can provide. In cruise flight the onflow velocity is higher than for takeoff. This leads to higher forces generated at the VTP which would allow a reduction of the VTP area. The conclusion is that the VTP size is determined by a rarely occurring failure case. In this critical OEI case the rudder is highly deflected to achieve maximum side force but the high deflection also leads to partial separation on the rudder, reducing the maximum side force which can be generated. If this separation could be avoided or delayed, the required side force could be generated by a smaller VTP. In turn, this would lead to a reduction in drag and weight, which in turn would reduce fuel burn and emissions. One possible means to achieve this is the use of active flow control (AFC). With this the side force coefficient produced by the VTP can be increased without increasing its size by delaying flow separation to higher sideslip and/or rudder deflection angles.

In contrast to passive flow control using for example vortex generators, active flow control has the possibility to be turned on just when needed avoiding the drag of the fixed vortex generators. On the other hand a source of energy is needed for the AFC system which is not the case for a passive system. For this investigation, pressurized air is used as the energy source to drive actuators. Such devices are already used in a wide range of active flow control applications [e. g. 1, 2, 3]. Among different kinds of blowing techniques tangential blowing over the rudder is selected. For this investigation the jet exit is located at the end of the fin.

Tangential blowing has already been investigated experimentally and numerically in several studies but mainly for the use on wings [4, 5]. In contrast to the wing, the VTP has a smaller aspect ratio and a larger sweep angle. Therefore it has flow characteristics which are different from those of a typical wing, leading to a more three dimensional flow. Thus the results obtained for wings, which usually show a more two dimensional flow, might not be directly transferable. Concerning AFC application to the VTP, some recent studies were done at NASA and Boeing with a mainly experimental approach [2, 6-9]. However, for these studies different kinds of actuators were used, namely synthetic jets and sweeping jets, which lead to a different interaction with the flow field. Some numerical investigations were done using the flow solver Overflow [3, 10] which was developed at NASA and Boeing and uses multiple overset structured grids. Substantial computational resources were invested to demonstrate the ability to simulate sweeping jets at a three dimensional vertical tailplane.

In the study presented here a different flow solver, namely the DLR Tau code with unstructured grids, as well as a different kind of actuator will be used. In addition, no study was found in which the underlying mechanism driving the variable effectivity of different slot configurations along the VTP span with tangential blowing was investigated.

Numerical work preceding the studies presented herein focused on the sensitivities of different active flow control parameters using a 2D VTP airfoil [11]. The current study extends this to 3D. This allows examining effects in spanwise direction for which a promising slot distribution along the span shall be found. The intention is to increase the side force coefficient while at the same time trying to increase the efficiency by using a low momentum coefficient. Therefore, starting from a continuous slot, the variation of the width of discrete slots and with this the size of the gap between them as well as the variation of the number of slots is the focus of the current work. In addition the velocity of the constant blowing jet is varied. The effects of blowing and the evolving vortex systems are studied as well as their impact on the side force coefficient. Since a complete 3D VTP would require a large mesh resulting in very high calculation time, a $2.5 \mathrm{D}$ geometry is used. It has an infinite span and a constant chord length but incorporates the sweep angle of the VTP. This setup allows for the investigation of a large number of configurations.

\section{GEOMETRY SETUP}

Due to the requirement for symmetrical behavior, the VTP usually has a symmetric airfoil. The NACA 63A010, which is a transonic airfoil suited for a conventional transport aircraft, is selected for this study. The hingeline, separating the non-moving fin and the deflectable rudder, is located at $67 \%$ chord length. Since this is a 2.5D approach, the chord length is constant along the span. At $44^{\circ}$ the sweep angle is similar to that of a VTP leading edge (cf. Fig. 1). The rudder deflection angle of $30^{\circ}$ perpendicular to the hinge line is selected due to the occurrence of notable flow separation for these conditions and was an outcome of the preceding $2 \mathrm{D}$ investigations. The side slip angle, analogous to the angle of attack for an aircraft wing, is zero for all investigations. The geometry is scaled by about $1: 11$ to correspond to a common wind tunnel scale and to make the results easier to compare with other studies.

A slot is integrated in the airfoil geometry at the end of the fin as shown in Fig. 2 to allow for tangential blowing. The slot height $h_{\text {Slot }}$ is 0.0006 of the chord length leading to a relatively thin slot. This height was also used for the preceding 2D investigations [11]. A part of the slot is modelled for numerical reasons [12]. The slot length $\mathrm{l}_{\text {Slot }}$ corresponds to 20 times its height and is chosen so that a developed pipe flow is established at the outlet. A small step is located aft of the slot towards the rudder shoulder, which would be expected for a realistic 3D design as well, if only due to material thickness. 


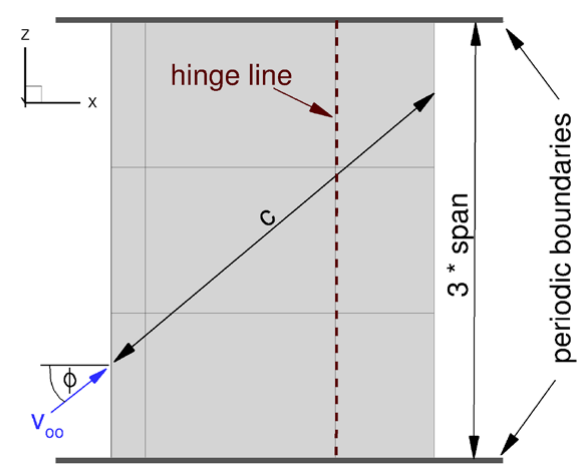

Fig. 1 Top view of the VTP section showing three times the span of the 2.5D computational model; c: chord length, $\phi$ : sweep angle; $v_{\infty}$ : onset flow velocity

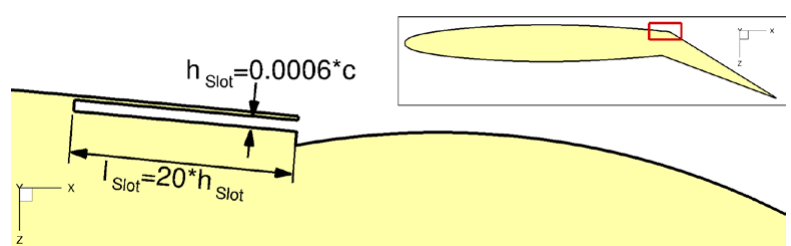

Fig. 2 Detail of the blowing slot geometry; $\mathrm{h}_{\text {Slot }}$ slot height, c: airfoil chord length, $\mathrm{l}_{\text {Slot }}$ slot length

The initial geometry uses a continuous slot. Further configurations are obtained with several discrete slots in spanwise direction. Their number, width and the gap size in-between are varied. One example is shown in Fig. 3. The left and right spanwise boundaries of the calculated section are defined as periodic boundaries as shown in Fig. 1. This leads to the simulation of a swept wing-type geometry with infinite span. The section of the span which is calculated is kept constant for the complete investigation. The width of the actuators is selected to enable an even spacing along the span for a given number of actuators. A list of all cases investigated is given in Tab. 1.

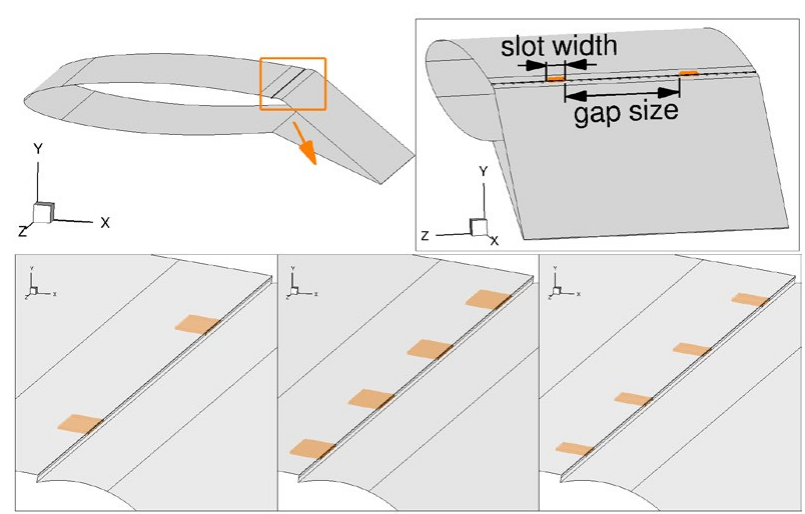

Fig. 3 2.5D VTP section with a detail view of three different slot configurations on the bottom: left: 2 slots, mid: 4 slots with same slot width, right: 4 slots with halved slot width; top right figure: aft view of the section

\begin{tabular}{|c|c|c|}
\hline $\begin{array}{c}\text { Number } \\
\text { of slots N }\end{array}$ & $\begin{array}{c}\text { Slot width w } \\
\text { per unit span }\end{array}$ & $\begin{array}{c}\text { Gap size } \\
\text { between slots g } \\
\text { per unit span }\end{array}$ \\
\hline 1 & 0.500 & 0.500 \\
\hline 1 & 0.250 & 0.750 \\
\hline 1 & 0.200 & 0.800 \\
\hline 1 & 0.125 & 0.875 \\
\hline 1 & 0.063 & 0.937 \\
\hline 1 & 0.031 & 0.969 \\
\hline \hline 2 & 0.438 & 0.063 \\
\hline 2 & 0.375 & 0.125 \\
\hline 2 & 0.250 & 0.250 \\
\hline 2 & 0.188 & 0.313 \\
\hline 2 & 0.125 & 0.375 \\
\hline 2 & 0.063 & 0.438 \\
\hline 2 & 0.031 & 0.469 \\
\hline \hline 3 & 0.125 & 0.209 \\
\hline 3 & 0.063 & 0.271 \\
\hline 3 & 0.031 & 0.302 \\
\hline \hline 4 & 0.125 & 0.125 \\
\hline 4 & 0.063 & 0.188 \\
\hline 4 & 0.031 & 0.218 \\
\hline \hline 5 & 0.125 & 0.075 \\
\hline 5 & 0.063 & 0.137 \\
\hline 5 & 0.031 & 0.169 \\
\hline \hline 6 & 0.063 & 0.104 \\
\hline \hline 8 & 0.063 & 0.063 \\
\hline 8 & 0.031 & 0.094 \\
\hline \hline 10 & 0.063 & 0.037 \\
\hline 10 & 0.031 & 0.069 \\
\hline
\end{tabular}

Tab. 1 List of all slot variations investigated

\section{MESH GENERATION}

For the mesh generation the commercial software Centaur by CentaurSoft is used [13]. It creates hybrid meshes. The surface mesh consists of triangles or quadrilaterals. The near-surface mesh is filled with prisms or hexahedrons and the remainder of the flow field with tetrahedrons. The slots themselves and also the rudder are meshed with quadrilaterals or hexahedrons wherever possible. In addition, the flow field behind and above the rudder is discretized with hexahedrons which are less dissipative and should lead to a better preservation of the flow quantities. In this area separated flow for the cases without or insufficient blowing and/or vortices due to the blowing jets is expected. An impression of the mesh in the vicinity of the vertical tailplane section is given in Fig 4.

Immediately aft of the slot the structured hexahedron mesh layers are reduced by the mesh generator in the corner of the step before growing again as shown in Fig. 5 a) and with a more detailed view in Fig. 5 b). Here some tetrahedrons must be used instead of the 
hexahedrons. This area is kept as small as possible by a rapid growth of the number of layers on the rudder shoulder.

Some aspects of mesh dependence were studied in a preliminary investigation described in [11] for a 2D case and were transferred to the current 2.5D mesh. The farfield extends 100 times the VTP chord length in $\mathrm{x}$ - and $\mathrm{y}$-direction. The overall number of mesh points is about 7 million resulting from 50 prism layers and a target dimensionless wall distance of $\mathrm{y}^{+}=0.5$. The relatively small $\mathrm{y}^{+}$improves the capturing of the flow close to the surface especially in the region of the jet exit nozzle and on the rudder.

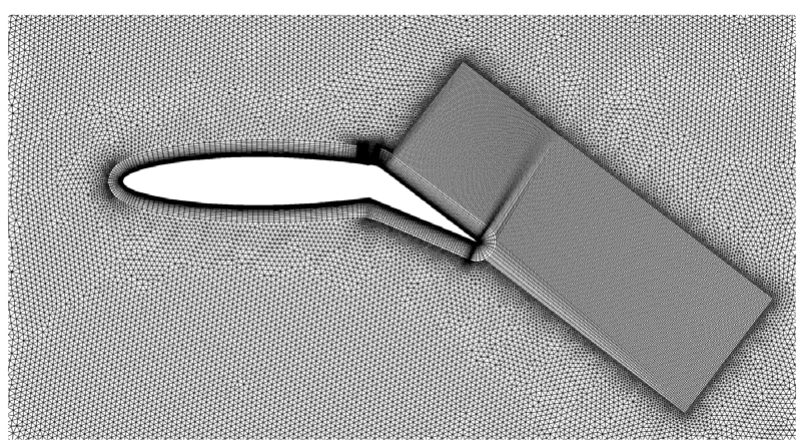

Fig. 4 Mesh with hexahedron field block refinement

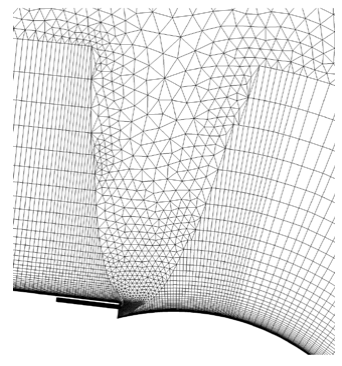

a) Mesh in the vicinity of the slot

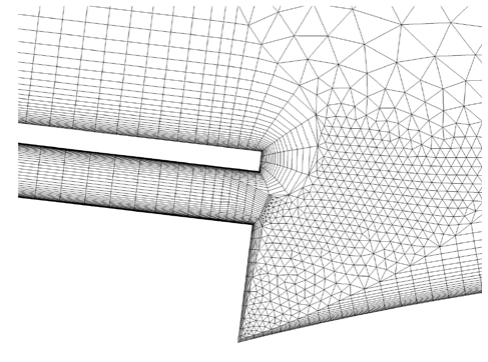

b) Zoom into the mesh at the slot exit
Fig. 5 Details of the mesh

\section{FLOW SIMULATION}

The flow computations were carried out with the flow solver TAU, release 2015.1.0, developed by DLR (Deutsches Zentrum für Luft- und Raumfahrt, German Aerospace Center) [14]. The TAU software solves the Reynolds-averaged Navier-Stokes (RANS) equations or alternatively the Euler equations in two or three dimensions on unstructured and hybrid grids.

For the current investigation compressible RANS calculations are performed. The spatial discretization is realized by a finite volume method. The temporal discretization is achieved using a semi-implicit Backward-Euler scheme with the linear LUSGS (LowerUpper Symmetric Gauss-Seidel) solver. The inviscid flux discretization type for the first stage of iterations is a first order upwind scheme, thereafter switching to the central scheme introduced by Jameson. A matrix dissipation scheme is employed for low numerical dissipation. For convergence acceleration a 3w multigrid scheme is used.

The viscous, fully turbulent RANS calculations were performed using the turbulence model of Spalart and Allmaras [15] enhanced with a vortical and rotational flow correction (SARC) based on the approach of Spalart-Shur [16]. For circulation control airfoils it was shown that this turbulence model leads to good results for flows with high streamline curvature [17]. For the calculations with blowing activated, an actuation boundary condition is specified at the upstream wall of the slot (cf. Fig. 2) to inject the jet flow into the flow domain. For this boundary condition a specification of jet velocity and density is necessary, with the latter assumed to be identical to the value of the flow in the farfield. Steady RANS calculations are performed with a constant blowing jet.

When comparing the results of the flow simulations, the dimensionless momentum coefficient $\mathrm{C}_{\mu}$ is used. It is defined as:

(1) $C_{\mu}=\frac{v_{j}^{2} * \rho_{j}^{*} A_{j}}{0.5 * \rho_{\infty} * v_{\infty}^{2} * A_{r e f}}$

where $\dot{\mathrm{m}}_{\mathrm{j}}=\mathrm{v}_{\mathrm{j}}{ }^{*} \rho_{\mathrm{j}}{ }^{*} \mathrm{~A}_{\mathrm{j}}$ is the mass flow rate of the jet through the actuator slot with the jet velocity $v_{j}$, the jet density $\rho_{j}$ and the area of the slot exit $A_{j}$. The variables $\rho_{\infty}$ and $v_{\infty}$ are the density and velocity of the onset flow in the farfield, $A_{\text {ref }}$ is the reference area of the model used.

For the flow simulations a Reynolds number of $\mathrm{Re}=2.24$ $\mathrm{x} 10^{6}$ based on the VTP airfoil chord length is used and a Mach number of $M=0.2$. In Tab. 1 an overview of all the geometries is given. The calculations are performed for each of the geometries for three different jet velocities which are $169 \mathrm{~m} / \mathrm{s}, 207 \mathrm{~m} / \mathrm{s}$ and $239 \mathrm{~m} / \mathrm{s}$. The convergence of the calculations is dependent on the momentum coefficient used and the ability to reduce the areas of separated flow. Without blowing the rudder is fully separated leading to an oscillating convergence. The calculations with the main jet velocity for this study $\left(v_{\mathrm{j}}=207 \mathrm{~m} / \mathrm{s}\right)$ as well as the calculations with differing jet velocities but $C_{\mu} \geq 0.5$ resulted in a standard deviation of the side force coefficient below $10^{-4}$ and a standard deviation of the drag coefficient below $10^{-5}$ if averaged over the last 3000 iterations. For lower and higher jet velocities the convergence of the side force and drag coefficients was slightly worse in the case of low momentum coefficients. The worst case showed a standard deviation of the side force coefficient of about $1.5 \%$ of its mean value. 


\section{RESULTS}

In this chapter results are presented initially for the case without blowing and for the continuous slot configuration. In a next step discrete slots are introduced and their effect on the side force coefficients is shown and discussed.

\subsection{Baseline Flow and Continuous Slot}

The largest slot width in spanwise direction can be obtained with a continuous slot. Since the slot height is fixed in this investigation, this leads to the largest slot exit area and thus to the largest mass flow rate for a given jet velocity. Therefore the side force coefficients obtained here are used as a reference to judge the effectivity of the subsequently performed slot variations.

To demonstrate the effect of the activation of the AFC, the results for the calculation without blowing are used as a reference. Without flow control the flow on the rudder is mostly separated for the rudder deflection angle selected. This is visualized in Fig. 6 a) where the velocity profiles just behind the rudder shoulder show an inflection point and reversed flow.

In a next step the momentum coefficient is increased gradually by increasing the jet velocity. At a momentum coefficient of $\mathrm{C}_{\mu}=1 \%$ the flow on the rudder is fully attached. In Fig. 6 b) the velocity profiles for this $C_{\mu}$ near the rudder shoulder are presented, showing the effect of the jet blowing tangentially over the surface. The jet velocity is higher than the surrounding local flow velocity. This leads to an increase of the flow velocity near the surface and to the bump seen in the velocity profile.

With increasing jet velocity or increasing $C_{\mu}$ the side force coefficient $C_{Y}$ increases and the drag coefficient $C_{D}$ decreases as shown in Fig. 7. The depicted increment in $C_{Y}$ and $C_{D}$ is the difference to the value obtained for the calculation without blowing $\left(C_{\mu}=0\right)$. The force coefficients are obtained by integrating the surface pressure and friction forces excluding the most downstream face of the slot where the jet boundary condition is set. With increasing $\mathrm{C}_{\mu}$ the energy introduced by the jet close to the rudder surface increases. Due to this the flow can better sustain the adverse pressure gradient further downstream on the rudder and the area of separated flow is reduced. Beyond $C_{\mu}=1 \%$, where the flow is fully attached up to the rudder trailing edge, $\mathrm{C}_{\mathrm{Y}}$ increases further but the efficiency of the blowing reduces. In this region for the same amount of additional $\mathrm{C}_{\mu}$ a smaller increase in $\mathrm{C}_{\mathrm{Y}}$ is obtained. The decrease in $\mathrm{C}_{\mathrm{D}}$ is due to the reduction of the separation on the rudder leading to a decrease in pressure drag. For $C_{\mu}>1 \%$ the drag coefficient increases slightly after the flow is fully attached due to an increase in the friction drag.

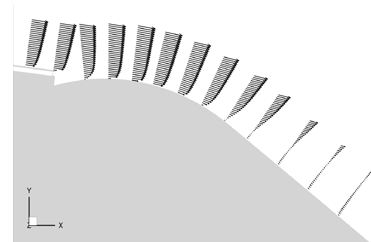

a) $\mathrm{C}_{\mu}=0$

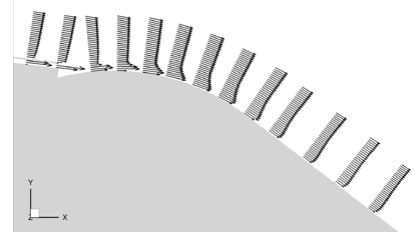

b) $\mathrm{C}_{\mu}=1 \%$

Fig. 6 Velocity vectors near the slot and on the rudder shoulder (length of vectors in a) twice that of b))

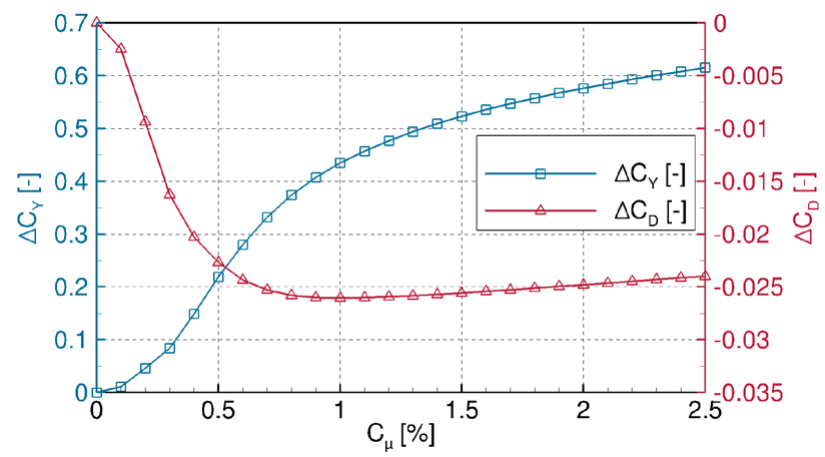

Fig. 7 Side force and drag coefficient increment over a variation of the momentum coefficient for the continuous slot

In Fig. 8 the surface pressure coefficient is shown for a selected momentum coefficient of $\mathrm{C}_{\mu}=0.8 \%$. This $\mathrm{C}_{\mu}$ is smaller than needed for the flow to remain attached up to the trailing edge. The two suction peaks at the fin leading edge and at the rudder shoulder are clearly visible. The peaks increase as the rudder flow separation is reduced. The stream traces on the fin visualize the high sweep angle of the incoming flow. On the rudder the flow does not follow this direction but is turned in the direction of the blowing jet which is perpendicular to the hinge-line. This effect increases as the momentum coefficient is increased. Since $C_{\mu}$ is not large enough, separated flow indicated by stream traces oriented in spanwise direction is visible towards the trailing edge.
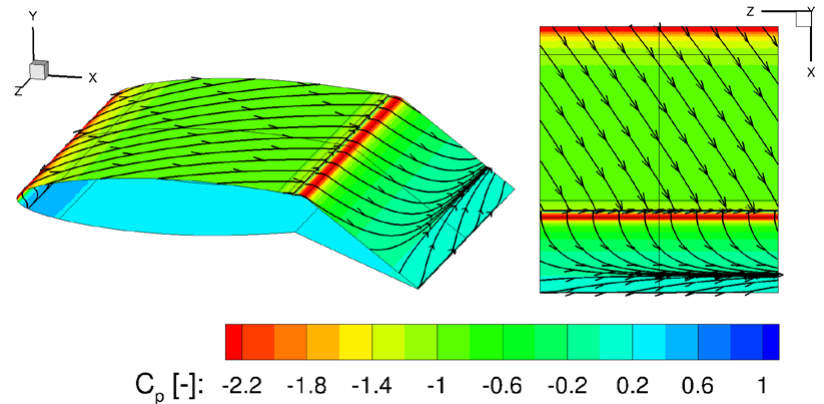

Fig. 8 Surface pressure coefficient $C_{p}$ and stream traces for the continuous slot, $\mathrm{C}_{\mu}=0.8 \%$; twice the calculated VTP section in spanwise direction is shown; Left: view from top aft, right: top view with the flow coming from the top 


\subsection{Discrete Slot Variations}

Using the continuous slot results as a reference, the area of the slot and its length in spanwise direction is now reduced. For the comparison of the results, three different jet velocities are investigated. These are selected based on the results for the continuous slot and correspond to momentum coefficients obtained there with $1 \%, 1.5 \%$ and $2 \%$. The respective jet velocities $\mathrm{v}_{\mathrm{j}}$ are: $169 \mathrm{~m} / \mathrm{s}, 207 \mathrm{~m} / \mathrm{s}$ and $239 \mathrm{~m} / \mathrm{s}$. Keeping $\mathrm{C}_{\mu}$ constant may not lead to a meaningful comparison for this investigation since this would lead to a very small jet velocity for slots with large width compared to small slots where the jet velocity would be very high and could also exceed the speed of sound. Apart from the jet velocity the number of the slots $\mathrm{N}$ and the slot width $\mathrm{w}$ are varied. Since the span is kept constant, the length of the gap between the slots results from the variation of the two former parameters.

\subsubsection{Flow Effects with Discrete Slots}

Replacing the continuous slot with several discrete slots changes the flow topology on the rudder. With blowing from the discrete slots activated, longitudinal vortices are created downstream of the rudder shoulder. These vortices are counter-rotating and are depicted for one selected case in Fig. 9. Field slices on the rudder are colored for the visualization of the vortices with $\omega_{x}$, the rotation direction in x-direction. From the observer point of view the blue vortices with negative $\omega_{\mathrm{x}}$, which are on the right of the vortex pair, are rotating clockwise and the red ones to their left rotate counter-clockwise.

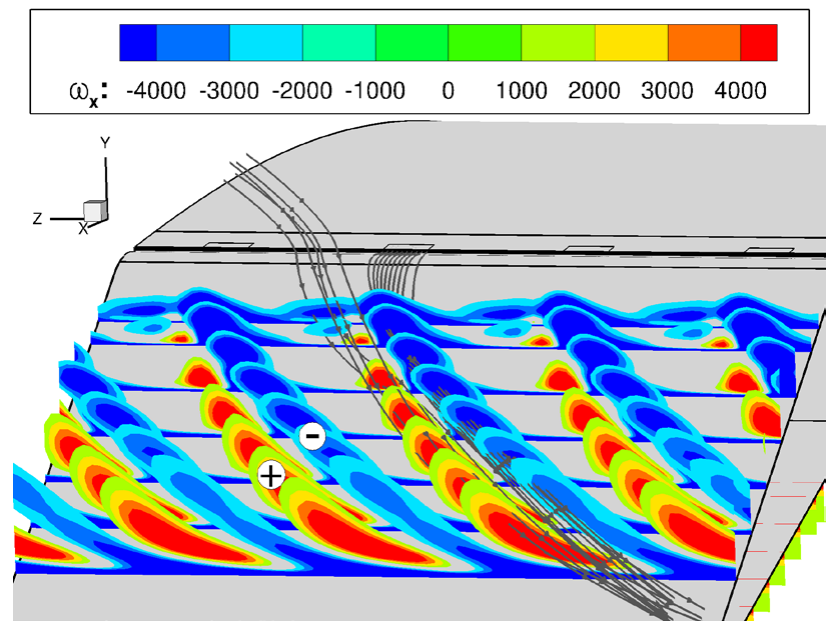

Fig. 9 Stream traces and field slices showing the vortices $\left(\mathrm{N}=4\right.$, w/span $\left.=0.063, \mathrm{v}_{\mathrm{j}}=239 \mathrm{~m} / \mathrm{s}, \mathrm{C}_{\mu}=0.5 \%\right)$

Two effects make it possible for the vortices to develop. The first one is that the slot has a discrete length creating a jet with a finite extension in spanwise direction. The second effect is that the actuator blowing direction has an angle relative to the incoming flow. The angle of the incoming flow is $44^{\circ}$ due to the sweep angle of the vertical tailplane section. This can be seen in Fig. 8 on the right side. The blowing direction of the jets is perpendicular to the rudder hinge-line. A close-up of the stream traces in a region where an actuator is placed is visualized in Fig. 10 a) where the view is from the top onto the slot and the rudder shoulder. The incoming flow from the fin attaches to the rudder shoulder if at this spanwise section no jet is blowing. Such parts only exist as long as the slots have a finite extension in spanwise direction. This can be seen in Fig. 10 b) where the stream traces of the flow from the fin get close to the rudder surface. Due to the inclination angle of this flow it meets the jet, as can be seen in the top view. It can to some extend deflect the jet. However, the jet has a higher velocity than the surrounding flow and is also attached to the rudder surface in this area. Thus the main flow cannot pass below the jet and bypasses it by going over it. This leads to some shearing between the two flow layers which induces a rotation leading to the blue vortex in Fig. 9 on the right side of the vortex pair. On its upwind side, where it rotates away from the surface, the counterrotating red vortex is induced with a positive rotation direction.

In the upwind region between the two vortices fluid is transported away from the surface, which is locally disadvantageous as this weakens the flow. On the other side of each vortex this is different. Here the rotation direction is towards the surface. This adds energy to the flow in this region which has an advantageous effect stabilizing the boundary layer. This helps to keep the flow on the rudder attached also in the areas between the jets. The generation of the vortices and their efficiency is dependent on the slot width and number of slots as well as on the jet velocity. Results for this parameter variation are presented in the next section.

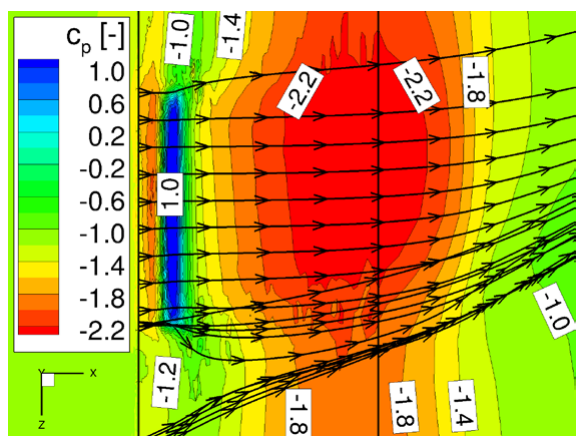

a) Top view

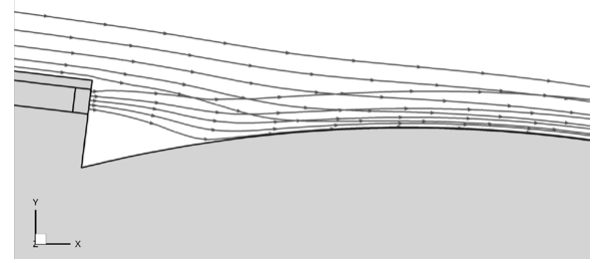

b) Side view

Fig. 10 View of the slot and part of the rudder shoulder; the stream traces shown are the same in both figures 


\subsubsection{Effects with Constant Jet Velocity}

In this section the influence on the side force coefficient is discussed for a study of a constant jet velocity while varying the number of slots and the slot width. From this it can be determined which slot configuration is most effective for a given jet velocity.

In Fig. 11 the increment of the side force coefficient $\Delta \mathrm{C}_{\mathrm{Y}}$ related to the value without blowing for the continuous slot versus the momentum coefficient $C_{\mu}$ is presented for a fixed jet velocity of $v_{j}=207 \mathrm{~m} / \mathrm{s}$. For one curve the number of slots is constant while the slot width is increased with increasing $\mathrm{C}_{\mu}$. For data points with the same $\mathrm{C}_{\mu}$ also the accumulated slot width is the same. The dotted line shows the results of the continuous slot while only the data point at $\mathrm{C}_{\mu}=1.5 \%$ on this curve is that for the considered jet velocity. Since the slot area is larger for the continuous slot than for the discrete slots, the jet velocity has to be smaller for the same $\mathrm{C}_{\mu}$.

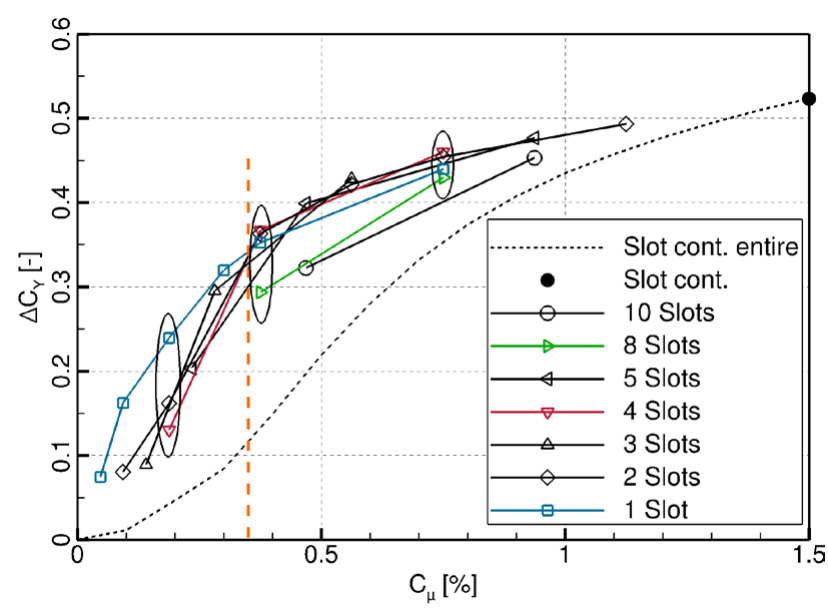

Fig. 11 Variation of $C_{Y}$ versus $C_{\mu}$ at $v_{j}=207 \mathrm{~m} / \mathrm{s}$

The figure reveals that for the same $C_{\mu}$ all results with discrete slots lead to a higher $\mathrm{C}_{\mathrm{Y}}$ than the continuous slot. Thereby the side force coefficient is increasing with increasing $\mathrm{C}_{\mu}$. In general three different regions with different effects can be observed and are discussed in the following sections.

\subsubsection{Region $\mathrm{C}_{\mu}<0.35 \%$}

The end of this region is marked in Fig. 11 with the vertical dashed line at $\mathrm{C}_{\mu}=0.35 \%$. Here the momentum coefficient is rather small. In this region the highest side force coefficient is obtained by a single slot and reduces when increasing the number of slots. A more detailed discussion of the flow effects will be exemplarily given for $\mathrm{C}_{\mu}=0.19 \%$. The results in consideration of one, two and four slots are encircled in Fig. 11. The jet velocity is constant for the results shown in this figure. For the continuous slot with its large slot area and at this velocity
$\mathrm{C}_{\mu}=1.5 \%$ results. Compared to this value the results at $\mathrm{C}_{\mu}=0.19 \%$ have only $1 / 8$ of this momentum coefficient. Since the jet velocity is constant, this decrease in $\mathrm{C}_{\mu}$ results just from the reduction in the slot area by this factor according to Eq. (1). This means that the mass flow rate is reduced by this factor as well. However, reducing the mass flow rate by $1 / 8$ does not necessarily mean that the side force increment is also reduced by $1 / 8$. Especially for the one-slot configuration it is much larger than $1 / 8$ of the value for the continuous slot configuration.

The reason why the one-slot configuration leads to the highest increase of $C_{Y}$ in this region of small momentum coefficients can be seen in Fig. 12. For the configuration with one slot with a large width, compare Fig. 12 a), the vortices created over the rudder have a much larger diameter than for the configuration with four smaller slots shown in Fig. 12 b). The vortices introduce energy from the outer flow into the region close to the surface. This enables the flow to stay attached to the surface of the rudder in this region. With two or four slots the vortices are smaller. Therefore the amount of energy they can bring close to the surface is smaller and the flow close to the rudder separates earlier. In addition, the vortices of the one-slot configuration are closer to the surface for a larger length of the rudder chord and thus have an increased influence while the ones for the four slots move away from the surface quite quickly.

For the small $\mathrm{C}_{\mu}$ where the jet separates quite quickly from the surface, the induced vortex created by the incoming flow with an angle to the jet passes below the jet vortex.

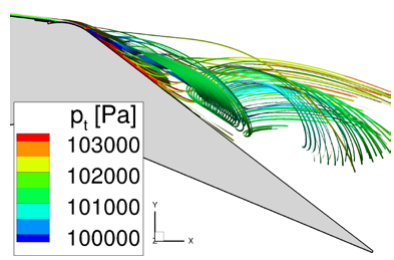

a) $\mathrm{N}=1$, w/span $=0.125$

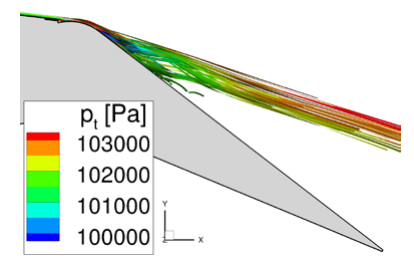

b) $\mathrm{N}=4$, w/span $=0.031$
Fig. 12 Side view of the rudder with field stream traces $\left(\mathrm{v}_{\mathrm{j}}=207 \mathrm{~m} / \mathrm{s}, \mathrm{C}_{\mu}=0.19 \%\right)$

In Fig. 13, Fig. 14 and Fig. 15 further visualization of the flow for the three slot configurations is shown. In part a) surface streamlines are depicted as well as the negative skin friction coefficient $\mathrm{C}_{\mathrm{fx}}$ in blue or darker color which gives an indication of reversed and separated flow. The top view of twice the vertical tailplane section is shown here leading to a doubling of the number of slots in this figure. In part b) field stream traces are depicted colored with total pressure with red representing a high and blue low total pressure. The levels are equal to those in the legend in Fig. 12. The field slices are colored with the vorticity about the $\mathrm{x}$-axis $\omega_{\mathrm{x}}$. Here just the blowing direction of the jet is in $\mathrm{x}$-direction and the vortices especially for this low momentum coefficient are more deflected in the direction of the outer flow. Nevertheless, 
this gives a good impression of the flow and the vortices over the rudder.

For the one large slot in Fig. 13 a large vortex pair is created which leads to an increased area of positive $\mathrm{C}_{\mathrm{fx}}$ that extends up to the trailing edge. However, due to the large gap between the slots a large area of the rudder is not affected by the vortices leading to separated flow in this region. In the case of the two or four smaller slots the area of positive $\mathrm{C}_{\mathrm{fx}}$ on the rudder is reduced. The vortices are weaker and further away from the surface leading to a reduced benefit of the mixing between the outer flow and the boundary layer. This, in sum, leads to a smaller side force coefficient than for the larger single slot. For this condition with low $\mathrm{C}_{\mu}$ it can be concluded that a smaller number of slots with greater lengths are beneficial compared to more but smaller slots.

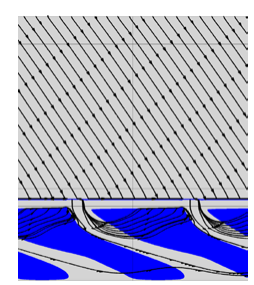

a) $\mathrm{C}_{\mathrm{fx}}<0$ and surface stream traces

Fig. $13 \mathrm{~N}=1$, w/span $=0.125, \mathrm{v}_{\mathrm{j}}=207 \mathrm{~m} / \mathrm{s}, \mathrm{C}_{\mu}=0.19 \%$, $\Delta \mathrm{C}_{\mathrm{Y}}=0.24$

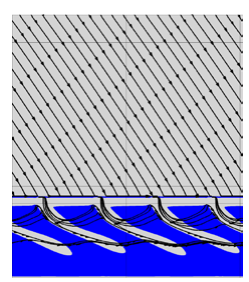

a) $\mathrm{C}_{\mathrm{fx}}<0$ and surface stream traces

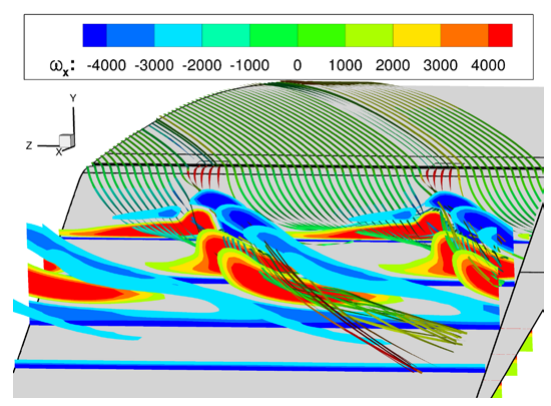

b) Stream traces $\left(\mathrm{p}_{\mathrm{t}}\right)$ and vortices $\left(\omega_{\mathrm{x}}\right)$

Fig. $14 \mathrm{~N}=2$, w/span $=0.063, \mathrm{v}_{\mathrm{j}}=207 \mathrm{~m} / \mathrm{s}, \mathrm{C}_{\mu}=0.19 \%$, $\Delta \mathrm{C}_{\mathrm{Y}}=0.16$

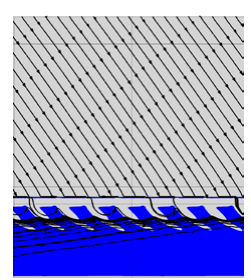

a) $\mathrm{C}_{\mathrm{fx}}<0$ and surface stream traces

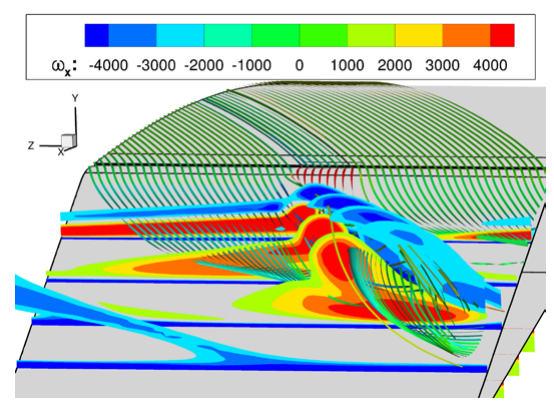

b) Stream traces $\left(\mathrm{p}_{\mathrm{t}}\right)$ and vortices $\left(\omega_{\mathrm{x}}\right)$

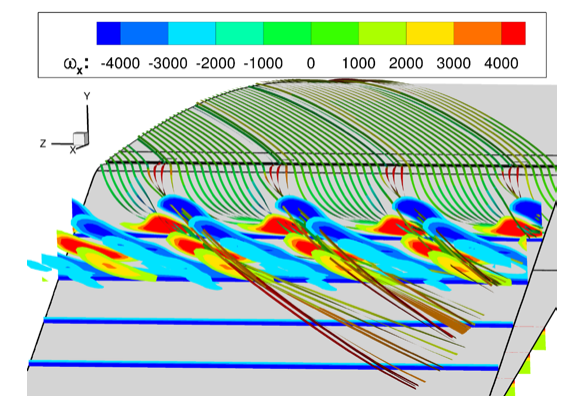

b) Stream traces $\left(p_{t}\right)$ and vortices $\left(\omega_{x}\right)$

Fig. $15 \mathrm{~N}=4$, w/span $=0.031, \mathrm{v}_{\mathrm{j}}=207 \mathrm{~m} / \mathrm{s}, \mathrm{C}_{\mu}=0.19 \%$, $\Delta \mathrm{C}_{\mathrm{Y}}=0.13$

\subsubsection{Region $0.35 \%<\mathrm{C}_{\mu}<0.9 \%$}

Due to the increased momentum coefficient or mass flow rate resulting from a larger slot length or an increased number of slots, higher values for the side force coefficient are obtained than seen for the smaller $C_{\mu}$. In this range of medium $C_{\mu}$ the efficiency of the one-slot configuration degrades or the efficiency of those with several slots increases. The results for the configurations with one to five slots are quite similar in this region of $\mathrm{C}_{\mu}$. However, with a large number of slots of eight or ten on the selected span a significant decrease in the $C_{Y}$ increment can be observed.

A second circle in Fig. 11 marks the results for $\mathrm{C}_{\mu}=$ $0.38 \%$. Compared to the circled results discussed before at $C_{\mu}=0.19 \%$ the area of the slots is doubled here. In addition, configurations with more slots are added where the slots are closer together, i.e. the gaps are reduced.

For this $C_{\mu}=0.38 \%$ the flow on the rudder is still not attached up to the trailing edge. Fig. 16 shows surface and field results for the configuration with one slot and Fig. 17 those with four slots which lead to the greatest increase in $\mathrm{C}_{\mathrm{Y}}$. Fig. 18 shows results for the configuration with eight slots which gives the smallest $C_{Y}$ for this $C_{\mu}$.

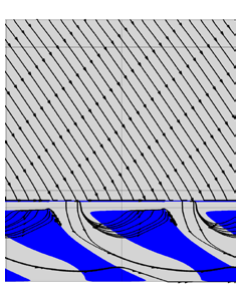

a) $\mathrm{C}_{\mathrm{fx}}<0$ and surface stream traces

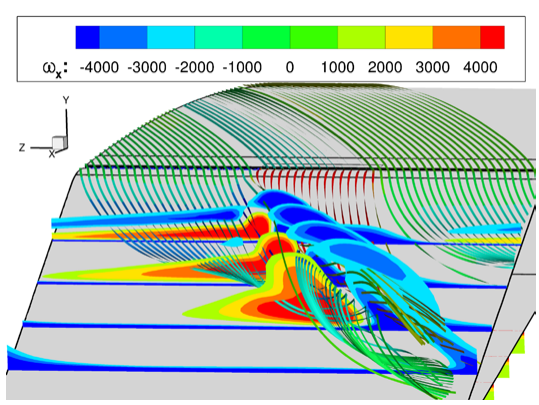

b) Stream traces $\left(\mathrm{p}_{\mathrm{t}}\right)$ and vortices $\left(\omega_{\mathrm{x}}\right)$

Fig. $16 \mathrm{~N}=1$, w/span $=0.250, \mathrm{v}_{\mathrm{j}}=207 \mathrm{~m} / \mathrm{s}, \mathrm{C}_{\mu}=0.38 \%$, $\Delta \mathrm{C}_{\mathrm{Y}}=0.35$

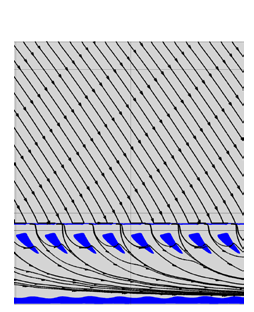

a) $\mathrm{C}_{\mathrm{fx}}<0$ and surface stream traces

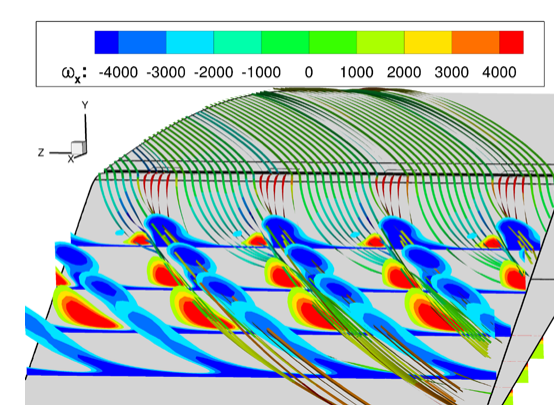

b) Stream traces $\left(p_{t}\right)$ and vortices $\left(\omega_{x}\right)$ 
Fig. $17 \mathrm{~N}=4$, w/span $=0.063, \mathrm{v}_{\mathrm{j}}=207 \mathrm{~m} / \mathrm{s}, \mathrm{C}_{\mu}=0.38 \%$, $\Delta \mathrm{C}_{\mathrm{Y}}=0.37$

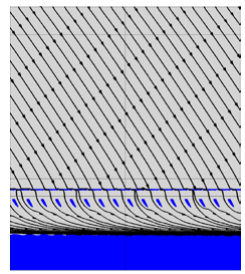

a) C $_{\mathrm{fx}}<0$ and surface stream traces

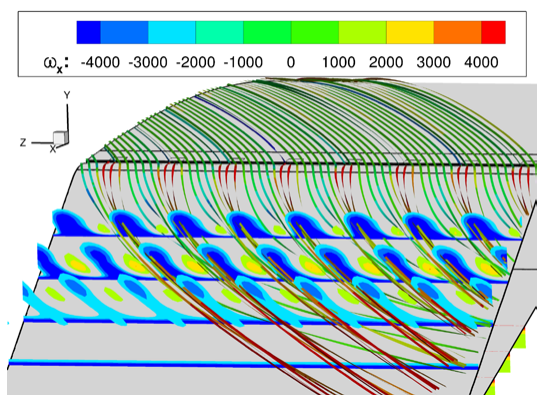

b) Stream traces $\left(\mathrm{p}_{\mathrm{t}}\right)$ and vortices $\left(\omega_{\mathrm{x}}\right)$

Fig. $18 \mathrm{~N}=8$, w/span $=0.031, \mathrm{v}_{\mathrm{j}}=207 \mathrm{~m} / \mathrm{s}, \mathrm{C}_{\mu}=0.38 \%$, $\Delta \mathrm{C}_{\mathrm{Y}}=0.29$

Two opposing effects can be observed here. For configurations with a large slot width and therefore also large gaps between the slots, the vortex system does not cover the entire span. Due to this, areas with separated flow exist between them, reducing the side force coefficient. On the other hand, a too dense slot spacing hinders the development of sufficiently large vortex structures, as can be observed in Fig. 18 for eight slots. Here the second vortex of each pair is relatively small and weak in its magnitude of $\omega_{\mathrm{x}}$.

Therefore the intermediate arrangement with four slots leads to the highest increase in the side force coefficient. The distance between the vortex pairs is such that the flow between them is attached. A small area of reversed flow is still visible near the trailing edge in Fig. 17 a). Here an increase in the jet velocity to $239 \mathrm{~m} / \mathrm{s}$ would add sufficient energy to the boundary layer to enable the flow to stay attached to the rudder up to the trailing edge.

Some small regions of reversed flow are visible between the blowing jets near the rudder shoulder where the separation would start without blowing. Since the vortices are not yet developed in this area, mainly the region along the span where the slots are positioned show attached flow. The jets widen towards the trailing edge and are then in combination with the fully developed vortex system able to cover the whole span further downstream on the rudder.

Increasing the accumulated slot area over the given span further by doubling it again leads to a slot area which is half that of the continuous slot. This would refer to the points circled in Fig. 11 at $C_{\mu}=0.75 \%$. The observations made before are applicable here as well. Due to an increased mass flow rate by larger or more slots, the flow on the rudder is attached up to the rudder trailing edge for most configurations. The differences in the $\mathrm{C}_{\mathrm{Y}}$ increment for the different configurations are reduced compared to the smaller momentum coefficients.

\subsubsection{Region $\mathrm{C}_{\mu}>\mathbf{0 . 9 \%}$}

For large momentum coefficients the differences in the values of the side force coefficient increments obtained decrease for the different slot configurations. The values are approaching those of the continuous slot case which is marked at $\mathrm{C}_{\mu}=1.5 \%$ in Fig. 11 . For this the maximum increase in $\mathrm{C}_{Y}$ for this jet velocity can be achieved but it also requires the largest mass flow rate making it less efficient.

\subsubsection{Variation of the Jet Velocity}

Increasing the jet velocity from $207 \mathrm{~m} / \mathrm{s}$ to $239 \mathrm{~m} / \mathrm{s}$ or decreasing it to $169 \mathrm{~m} / \mathrm{s}$ leads in principle to a shift of the curves shown in Fig. 11. For the higher jet velocity the gain in $\mathrm{C}_{\mathrm{Y}}$ is slightly increased in the same range of $\mathrm{C}_{\mu}$ and since higher $C_{\mu}$ values can be obtained, the achievable side force coefficient is also increased. The opposite is true for the lower jet velocity. The respective diagrams are shown in Fig. 19 and Fig. 20.

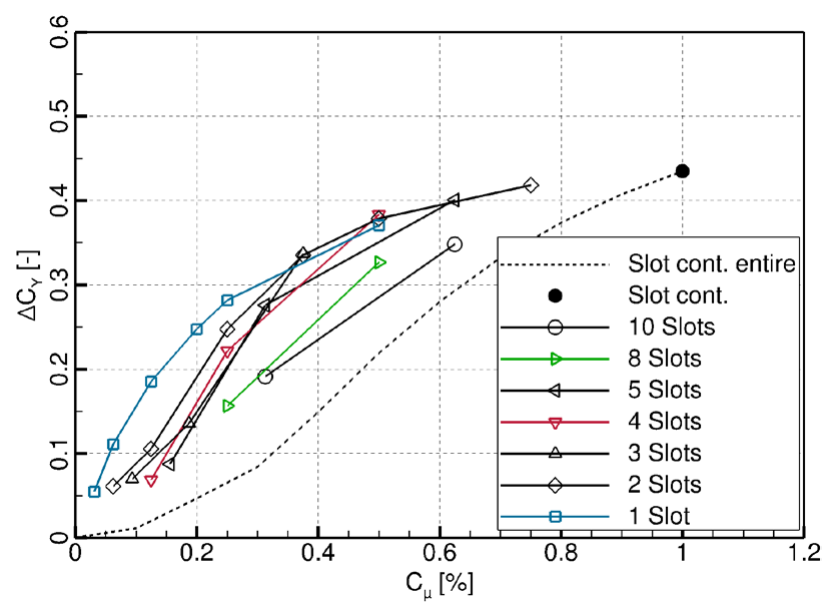

Fig. 19 Variation of $C_{Y}$ versus $C_{\mu}$ at $v_{j}=169 \mathrm{~m} / \mathrm{s}$

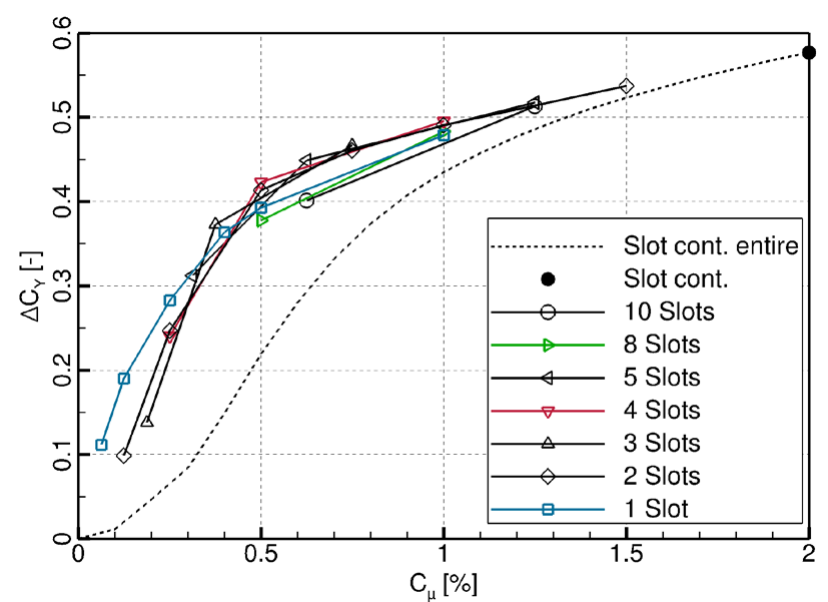

Fig. 20 Variation of $C_{Y}$ versus $C_{\mu}$ at $v_{j}=239 \mathrm{~m} / \mathrm{s}$

In addition it can be observed that for the different slot configurations at the same $C_{\mu}$ the obtained $C_{Y}$ is more similar for the case with the large jet velocity. For the smaller jet velocity the variation in the side force 
coefficient is much larger for the same momentum coefficient and the reduced efficiency of more but smaller slots even more noticeable.

\subsubsection{Effects with Constant Slot Width}

Until now the jet velocity was kept constant in one diagram. In this section the results are discussed under the viewpoint of a constant slot width. Selected is a slot width of w/span $=0.063$. With the slot width fixed, the number of slots $\mathrm{N}$ and with this also the gap between the slots $\mathrm{g}$ can be varied to judge how many slots are necessary to keep the flow on the rudder attached. The results are shown in Fig. 21. In addition, the effect of the jet velocity is monitored. Each data row consists of results for the three different jet velocities of $169 \mathrm{~m} / \mathrm{s}, 207 \mathrm{~m} / \mathrm{s}$ and 239 $\mathrm{m} / \mathrm{s}$. The smallest jet velocity leads to the lowest increase in $\mathrm{C}_{\mathrm{Y}}$ for each row of data.

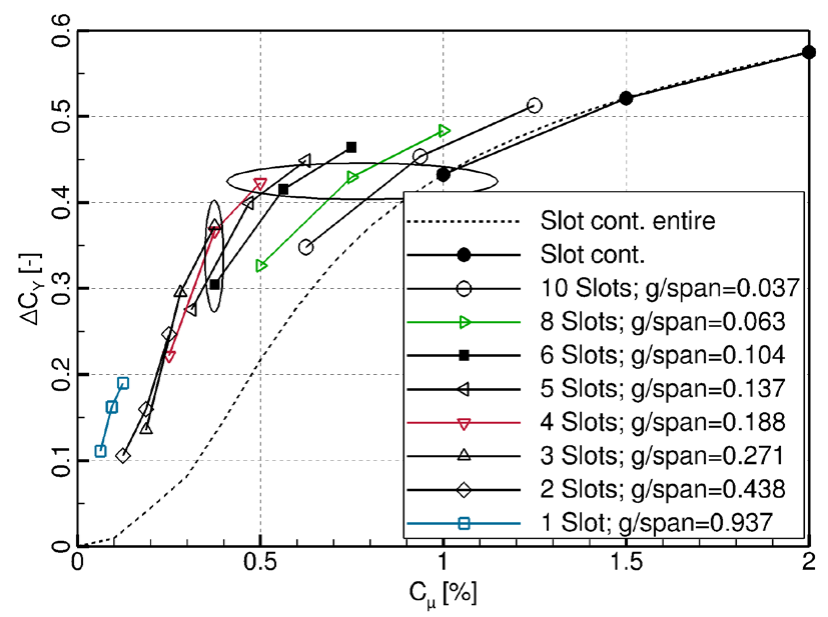

Fig. 21 Variation of $C_{Y}$ versus $C_{\mu}$ at a constant slot width $\mathrm{w} / \mathrm{span}=0.063$ and varying gap size $\mathrm{g}$

The discussion of the results is possible for constant $\mathrm{C}_{\mu}$ and for constant $\Delta \mathrm{C}_{\mathrm{Y}}$. Both will be done in the two following sections.

\subsubsection{Constant Momentum Coefficient}

At $\mathrm{C}_{\mu}=0.38 \%$ three results are circled and chosen for the subsequent discussion. For these the flow on the rudder is not yet completely attached. The areas with negative skin friction coefficient $\mathrm{C}_{\mathrm{fx}}$ are marked in Fig. 22 for these three results. In this figure twice the calculated span is shown.

The result for $\mathrm{N}=3$ has the lowest number of slots but the highest jet velocity and also the largest increase in $\Delta \mathrm{C}_{\mathrm{Y}}$. Here the flow is practically fully attached up to the trailing edge but larger areas of separated flow exist between the slots. The result with the largest number of slots $\mathrm{N}=6$ has the smallest jet velocity. The areas of reversed flow between the slots is very small but since the jet velocity is the lowest, not enough energy is added to the boundary layer making it more difficult to overcome the adverse pressure gradient leading finally to the separation of the flow from the rudder and the reduced gain in $\Delta \mathrm{C}_{\mathrm{Y}}$. The results for the configuration with $\mathrm{N}=4$ slots and medium slot velocity shows both effects: The separated regions between the jets close to the rudder shoulder and some separation near the trailing edge. However, these are less distinct than for the two other configurations and lead in the end to a $\Delta \mathrm{C}_{\mathrm{Y}}$ close to the one with $\mathrm{N}=3$.

In summary it can be stated that for the same $\mathrm{C}_{\mu}$ a lower number of slots with a higher jet velocity results in the largest increment in side force coefficient.
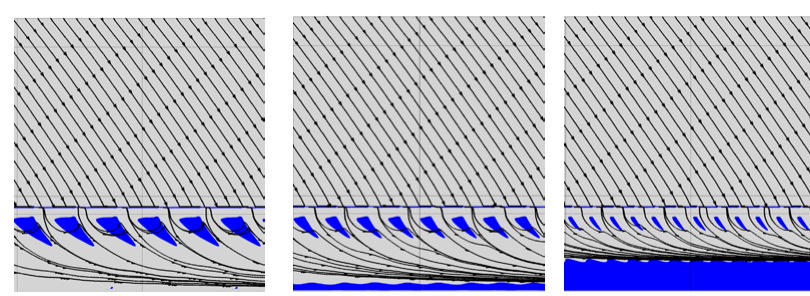
a) $\mathrm{N}=3$,
b) $\mathrm{N}=4$,
c) $\mathrm{N}=6$,
$\mathrm{v}_{\mathrm{j}}=239 \mathrm{~m} / \mathrm{s}$
$\mathrm{v}_{\mathrm{j}}=207 \mathrm{~m} / \mathrm{s}$
$\mathrm{v}_{\mathrm{j}}=169 \mathrm{~m} / \mathrm{s}$

Fig. 22 Top view onto the vertical tailplane section showing $\mathrm{C}_{\mathrm{fx}}<0$ and surface stream traces

$\left(\mathrm{w} / \mathrm{span}=0.063, \mathrm{C}_{\mu}=0.38 \%\right)$

\subsubsection{Constant $\Delta \mathrm{C}_{\mathbf{Y}}$}

In a second step those results are reviewed which lead to a similar increase in the side force coefficient. Now the horizontally oriented selection encircled in Fig. 21 for a $\Delta \mathrm{C}_{\mathrm{Y}}$ of about 0.42 are discussed. All configurations show attached flow up to the trailing edge but the size of the areas of separated flow between the jets close to the rudder shoulder differs. Although the same gain in $C_{Y}$ is obtained, the required momentum coefficient is quite different for each case.

The most effective configuration is the one with $\mathrm{N}=4$ and the highest jet velocity requiring a $\mathrm{C}_{\mu}=0.5 \%$. Compared to the continuous slot this means a large reduction of the momentum coefficient by $50 \%$. When considering the mass flow rate this would correspond to a reduction of $65 \%$ compared to the continuous slot.

For the configuration with four slots the spacing of the slots seems optimal to cover the span with the vortex system as shown in Fig. 23 a). Increasing the number of slots reduces the distance between them too much so that the vortex pairs cannot fully develop. For $\mathrm{N}=8$ this is shown in Fig. 23 b). For the continuous slot no vortices are present at all. 
With an increasing number of slots the accumulated slot area increases due to the constant width. On the other hand the jet velocity for the configurations with $\mathrm{N}=6$ and $\mathrm{N}=8$ slots is smaller than for the configuration with four slots and the smallest for the continuous slot. However, the increase in the slot area outweighs the velocity decrease, leading to an increase in $C_{\mu}$ for the configurations with more slots.

In summary it was found that the configuration with the highest jet velocity and the smaller number of slots leads to the most effective configuration.

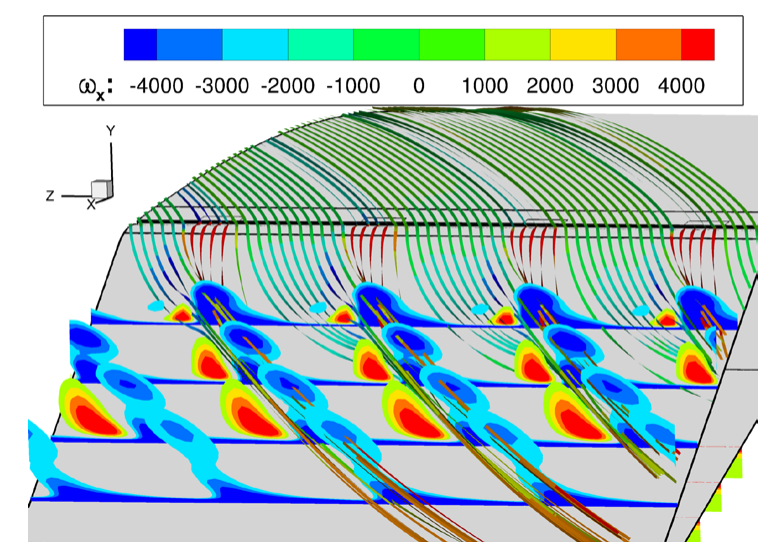

a) $\mathrm{N}=4, \mathrm{v}_{\mathrm{j}}=239 \mathrm{~m} / \mathrm{s}, \mathrm{C}_{\mu}=0.5, \Delta \mathrm{C}_{\mathrm{Y}}=0.42$

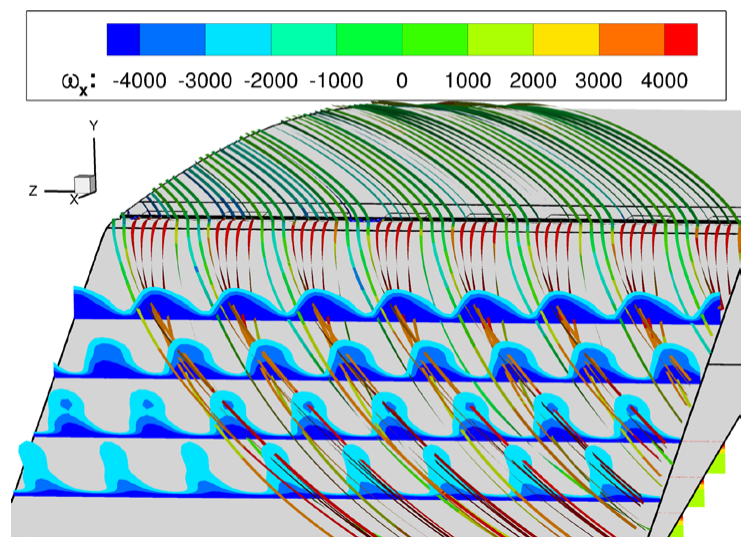

b) $\mathrm{N}=8, \mathrm{v}_{\mathrm{j}}=207 \mathrm{~m} / \mathrm{s}, \mathrm{C}_{\mu}=0.75, \Delta \mathrm{C}_{\mathrm{Y}}=0.43$

Fig. 23 Stream traces $\left(\mathrm{p}_{\mathrm{t}}\right)$ and vortices $\left(\omega_{\mathrm{x}}\right)$ when looking from the aft onto the rudder

\section{CONCLUSION}

For a 2.5D vertical tailplane tangential blowing over the shoulder of a deflected rudder is investigated in a numerical study. Starting with a configuration with a continuous slot extending over the whole span this slot is replaced by configurations with discrete slots. The number of slots, their width as well as the jet velocity is varied. The aim is to determine the largest increase in the side force coefficient with the lowest momentum coefficient.

Steady calculations are performed using the Reynoldsaveraged Navier-Stokes equations with the flow solver TAU. Without the tangential blowing the flow on the deflected rudder of the vertical tailplane is separated. With blowing activated and a sufficient jet velocity the flow could be held attached for the continuous slot. Using discrete slots additional effects were observed which make these configurations more efficient.

It could be shown that for the discrete slots a pair of counter-rotating vortices is created if the slots have sufficient space between them. In addition to the energy fed to the boundary layer by the jet itself this leads to an increased mixing of the outer and near-surface flow, also increasing the energy of the boundary layer. Due to this the separated flow could be reduced or even avoided.

For small momentum coefficients $\mathrm{C}_{\mu}<0.3 \%$ a defined increment in $\mathrm{C}_{\mu}$ leads to a relatively large increase in the side force coefficient $C_{Y}$. However, the flow on the rudder is not fully attached so the gain in $\mathrm{C}_{\mathrm{Y}}$ is limited. By increasing the momentum coefficient the separation on the rudder can be reduced but the increase in $C_{Y}$ per $\Delta \mathrm{C}_{\mu}$ decreases.

For $\mathrm{C}_{\mu}<0.3 \%$ the configuration with one slot leads to the largest increase in $\mathrm{C}_{\mathrm{Y}}$ because the created vortex pair is the largest. With increasing $C_{\mu}$ at constant jet velocity the size of the slot becomes larger than necessary to create a sufficiently large vortex pair. Since the gap between the slots is quite large as well, areas of separation can develop in-between them. On the other hand having a too small gap between the slots is also not beneficial as the development of the vortex system is suppressed. An optimal configuration was found for a number of slots of four on the defined span with a slot width per span of 0.063 and a gap between them of 0.188 , i.e. a ratio of $1: 4$ between slot and gap width. With $\mathrm{C}_{\mu}=0.5 \%$ attached flow could be achieved on the rudder. This amounts to half the $\mathrm{C}_{\mu}$ required for full flow attachment with the continuous slot.

In general it can be stated that for a constant slot width the configuration which combines a small number of slots with a large jet velocity is most effective for a similar increase in side force coefficient. One interesting next step would now be the transfer of these results to a complete three dimensional vertical tailplane where additional 3D effects will likely have an influence.

\section{REFERENCES}

[1] Seifert, A., Tilmann, C. P.: Control of flow separation for fixed wing airfoil applications, In: Bonnet, J.-P., Anthoine, J. (eds.) Flow Control: 
Fundamentals, Advances and Applications. Von Karman Institute for Fluid Dynamics Lecture Series 2009-02, Rhode Saint Genese, Belgium (2009)

[2] Andino, M. Y., Lin, J. C., Washburn, A. E., Whalen, E. A., Graff, E. C., Wygnanski, I. J.: Flow Separation Control on a Full-Scale Vertical Tail Model using Sweeping Jet Actuators. AIAA Paper 2015-0785 (2015)

[3] Childs, R. E., Stremel, P. M., Garcia, J. A., Heineck, J. T. , Kushner, L. K., Stormsk, B. L.: Simulation of Sweep-Jet Flow Control, Single Jet and Full Vertical Tail. AIAA Paper 2016-0569 (2016)

[4] Pfingsten, K. C., Radespiel, R.: Experimental and numerical investigation of a circulation control airfoil. AIAA Paper 2009-533 (2009)

[5] Burnazzi, M., Radespiel, R.: Design of a Droopnose Configuration for a Coanda Active Flap Application. AIAA Paper 2013-0487 (2013)

[6] Rathay, N. W., Boucher, M. J., Amitay, M., Whalen, E.: Performance Enhancement of a Vertical Stabilizer using Synthetic Jet Actuators: No Sideslip. AIAA Paper 2012-0071 (2012)

[7] Rathay, N. W., Boucher, M. J., Amitay, M., Whalen, E.: Performance Enhancement of a Vertical Stabilizer using Synthetic Jet Actuators: Non-zero Sideslip. AIAA Paper 2012-2657 (2012)

[8] Seele, R., Graft, E., Gharib, M.: Improving Rudder Effectiveness with Sweeping Jet Actuators. AIAA Paper 2012-3244 (2012)

[9] Lin, J. C., Andino, M. Y., Alexander, M. G., Whalen, E. A., Spoor, M. A., Tran, J. T., Wygnanski, I. J.: An Overview of Active Flow Control Enhanced Vertical Tail Technology Development. AIAA Paper 2016-0056 (2016)

[10] Shmilovich, A. and Yadlin, Y. and Whalen, E.: Computational Evaluation of Flow Control for Enhanced Control Authority of a Vertical Tail, AIAA Paper 2015-3311 (2015)

[11] Kröhnert, A.: Numerical Investigation of Tangential Blowing at the Rudder of a Vertical Tailplane Airfoil. AIAA Paper 2014-2143 (2014)

[12] Ciobaca, V.: Validation of Numerical Simulations for Separation Control on High-Lift Configurations, Ph.D. Thesis, DLR (German Aerospace Center) (2014)

[13] CentaurSoft: Centaur Hybrid Grid Generation System, http://www.centaursoft.com (2016). Accessed 26 August 2016.

[14] Gerhold, T.: Overview of the Hybrid RANS Code TAU. In: Kroll, N., Fassbender, J. (eds.) MEGAFLOW-Numerical Flow Simulation for Aircraft Design. Notes on Numerical Fluid Mechanics and Multidisciplinary Design, NNFM, Vol. 89, pp. 81-92. Springer, Heidelberg (2005)

[15] Spalart, P., Allmaras, S.: One-Equation Turbulence Model for Aerodynamic Flows. AIAA Paper 920439 (1992)

[16] Shur, M. L., Strelets, M. K., Travin, A. K., Spalart, P. R.: Turbulence Modeling in Rotating and Curved
Channels: Assessing the Spalart-Shur Correction. AIAA Journal, Vol. 38, No. 5, pp. 784-792 (2000)

[17] Pfingsten, K.-C., Jensch, C., Körber, K. W., Radespiel, R.: Numerical Simulation of the Flow around Circulation Control Airfoils. CEAS 2007377 (2007) 